Please do not remove this page

RMIT

UNIVERSITY

\title{
Estimating ridge topologies with high curvature for fingerprint authentication systems
}

Wang, Yi; Hu, Jiankun

https://researchrepository.rmit.edu.au/esploro/outputs/9921858995801341/filesAndLinks?institution=61RMIT_INST\&index=null

Wang, Y., \& Hu, J. (2007). Estimating ridge topologies with high curvature for fingerprint authentication systems. ICC 2007 Proceedings, 1179-1184. https://doi.org/10.1109/ICC.2007.200

Published Version: https://doi.org/10.1109/ICC.2007.200

Repository homepage: https://researchrepository.rmit.edu.au

(C) 2007 IEEE

Downloaded On 2023/04/26 20:13:49 +1000

Please do not remove this page 


\title{
Estimating Ridge Topologies with High Curvature for Fingerprint Authentication Systems
}

\author{
Yi Wang and Jiankun $\mathrm{Hu}$ \\ ${ }^{*}$ School of Computer Science and Information Technology \\ Royal Melbourne Institute of Technology, Melbourne, Australia 3001 \\ Email: $\{$ alice, jiankun\}@cs.rmit.edu.au
}

\begin{abstract}
An orientation model provides an analytical means for describing fingerprint ridge orientations. It can help in data storage and recovery as well as other possible communication applications related to biometric security. Since fingerprint ridge patterns often possess both smooth features and high curvature patterns, it is not easy to describe the overall topology with a single analytical model. A combination approach of different models is a way to address the problem. In this paper, we explore this topic by investigating local orientation models for estimating high curvature patterns in the singular regions. Our experimental results show that the resulting combination approach can improve the overall topology estimation and thus the end performance of a fingerprint authentication system.
\end{abstract}

\section{INTRODUCTION}

With the pervasive growth of Internet computing, it is highly desirable to have reliable safeguards for sensitive data and personal resources. Critical information of data transaction over the web can be encrypted using advanced cryptography methods and data hiding techniques. However, these security measures are not capable of validating the genuine users. This vulnerability has been evidenced by numerous spoofing and intrusion attacks. People are now more conscious than ever that the current password based authentication is just not secure enough.

On the other hand, everyone possesses permanent biometric traits that cannot be stolen or deceived. Thus much effort has come to deploy effective biometric based security mechanisms. Fingerprints are highly distinctive between individuals [1] and the acquisition device is user friendly and with a relatively low cost. These beneficial features have made it attractive for personal identification.

Estimating ridge orientations is essential for many fingerprint-based applications. For instance, it can be used to enhanced the fingerprint quality which helps to extract distinctive features. Most fingerprint ridge topologies are not overall smooth. They often encompass singular regions where the ridge flows are wrapped around a Singular Point (SP) with discontinuous manner.

Ridge pattern near a SP is often characterized by high curvature. This can result in a hard tradeoff for orientation estimation. For instance, a large estimation window may be beneficial for averaging noise but may also suppress ridge curvature especially in singular regions [2]. Therefore, post processing techniques, such as using hierarchical window size
[3] and weighted averaging method [4], were proposed in the literature.

Compared to the enhanced averaging methods, mathematical modeling can provide other functions in addition to refining the orientation estimation. A good orientation model can provide a comprehensive description of the subject, and thus enables discovery of the embedded features and use in other applications. If a mathematical model can be used to reconstruct the orientation field, it is only necessary to store and analyze the model coefficients. Since the number of coefficients is usually much less than the number of raw orientation data, it is an advantage for embedded applications where resource is often highly constrained. A mathematical model for fingerprint orientation may also have other potential applications for communication systems such as indexing and bio-cryptography.

Several orientation models have been proposed in the literature. Sherlock and Monro [5] describe orientation fields using the square root of a rational polynomial function with firstorder poles and zeros. However, the reconstructed orientation patterns are only for "ideal" ridge topologies.

Rao and Jain [11] discussed the linear system of firstorder differential equations for modeling texture flows. Some of the linear phase portraits resemble the singular patterns of fingerprints closely. However, the linear system can only accommodate at most one singular point (SP). Therefore, it is not suitable for describing the overall fingerprint orientation pattern where multiple SPs can be embedded. Nevertheless, the phase portrait analysis has inspired many other considerations including our ideas as will be shown later in this paper.

Yao [8] et. al. proposed a nonlinear expansion of Taylor series with linear constraints placed at singular points in order to insure the desired orientation patterns can be generated from the first-order phase portraits at SPs.

Zhou et. al. [7] combined a higher order polynomial model and a point-charge model for different regions. The polynomial function is used to describe the overall ridge topology and the point-charge model is used to correct the local description around singular points. A global weighing function is used to combine the different models with respect to the distance from each SP in the orientation field.

Since fingerprints often possess both smooth features in a global sense and steep changes in local regions, it is not easy to describe the overall topology with a single mathematical 
model. In this paper, we propose a new combination approach to address the problem.

We first derive an elliptic curve model from the linear system that can generate desired orientation patterns. The proposed elliptic model possesses advantages over the pointcharge model [7] and the nonlinear-constrained model [8]. This is because the point-charge model can represent at most one SP in a local region. Its performance degrades in cases when multiple SPs are geometrically crowded and have influence on each other. The elliptic curve model does not suffer from this problem as it can capture up to four SPs [9]. The constrained nonlinear optimization adds computational complexity to the nonlinear-constrained model. We show that a second order polynomial can approximate the elliptic curve model with little sacrificing in accuracy but great improvement in computational advantage.

The rest of the paper is organized as follows. Section II presents the proposed local orientation models. Section III investigates different combination approaches and compares their performance and impacts on fingerprint authentication systems. Finally, we conclude in Section IV.

\section{The Proposed Orientation Estimation}

Fingerprint orientation field (OF) depicts the dominant ridge orientation in lattice for a fingerprint image. Since only the orientation angle is of interest, the OF can be represented in the form of $\left\{\theta_{i j}\right\}$, where $\theta_{i j} \in[0, \pi)$ and $(i, j)$ are the lattice indices.

The local orientation $\theta_{i j}$ can be derived from the gradients of gray intensity change in a fingerprint image. The gradients are pointing to the highest variation of gray intensity. Therefore, the dominant gradient direction is perpendicular to the local ridge orientation which is in tangential to the ridge flows.

It is difficult to extract the dominant gradients at high curvature areas via the normal averaging process [10]. High curvature often appear near singular points (SP). Based on the ridge assembling behavior, SP can be classified into three basic topologies: whorl(twin loop), loop and delta [1]. Figure 1 shows some typical ridge patterns of these kinds.

From a geometric point of view, the whorl type pattern is very much like concentric circles, while the twin loop is similar to cubical parabolic hyperbola and the loop is of parabola shape. A research question naturally arises: is it possible to analyze the ridge orientation patterns with differential geometric theories?

The linear system of first-order differential equations can be written as:

$$
\begin{aligned}
& \dot{x}=F(x, y)=a x+b y+e \\
& \dot{y}=G(x, y)=c x+d y+f,
\end{aligned}
$$

where $\mathbf{x}=[x, y]^{T}$ is a vector in the two-dimensional space $\mathcal{R}^{2}$ and $\dot{\mathbf{x}}=\left[\dot{x}_{1}, \dot{y}_{2}\right]^{T}$ is the gradient vector of $\mathbf{x}$. The above equations can be represented with matrix notation:

$$
\dot{\mathbf{x}}=\mathbf{A x}+\mathbf{b}
$$

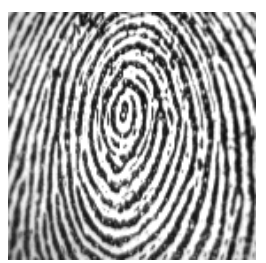

(a) Whorl

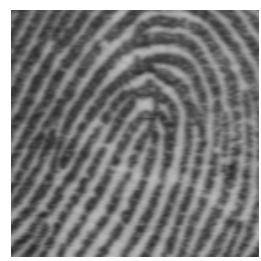

(c) Loop

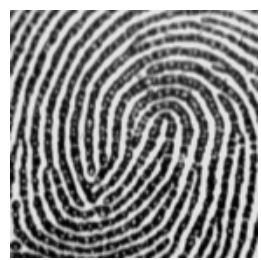

(b) Twin loop

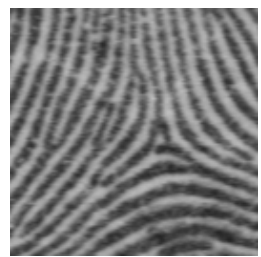

(d) Delta
Fig. 1. Ridge patterns with high curvature around SP.

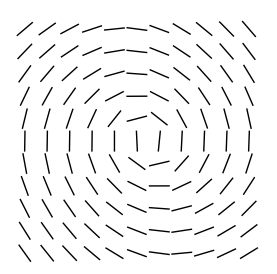

(a) Center

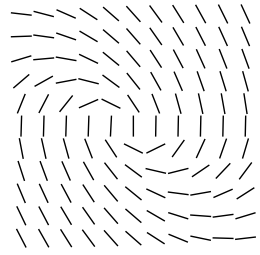

(b) Spiral

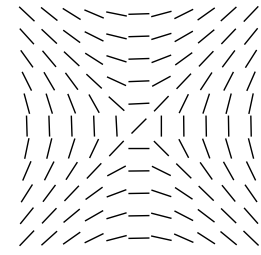

(c) Saddle
Fig. 2. Phase portraits generated from the linear system (1)

where $\mathbf{A}$ is the characteristic matrix, and $\mathbf{b}=[e, f]^{T}$ is the translational component of the system. If $|\mathbf{A}| \neq \mathbf{0}$, there is one SP at the origin. Otherwise, i.e. $|\mathbf{A}|=\mathbf{0}$, no SP is present in the local neighborhood [11]. Further, iff $|\mathbf{A}|<\mathbf{0}$, i.e. $a d<b c$, the linear system $\dot{\mathbf{x}}=\mathbf{A x}$ has a saddle-like SP at the origin as shown in Figure 2(c). However, positive $|\mathbf{A}|$ may result in several different flow patterns other than the saddle [12]. Two examples are shown in 2(a) and 2(b).

Comparing Figure 1 and 2, one can see that the centers and spirals resemble the whorl and twin-loop topologies quite well. Although the loop-type and delta-type SP can not find direct parts in the six flow patterns, we observe that they have strong resemblance to the portraits on the half-phases (phase angles divided by 2) derived from the center/spiral and saddle patterns respectively. Figure 3 displays the corresponding halfphase portraits generated from those shown in Figure 2. Note that variation, such as squeezing and rotation, of the patterns shown in the phase portraits can be achieved by scaling and reversing the elements in the characteristic matrix $\mathbf{A}$ and the translation vector $\mathbf{b}$.

In practice, the linear system (1) cannot be directly used to model ridge orientations. There are three main reasons. 


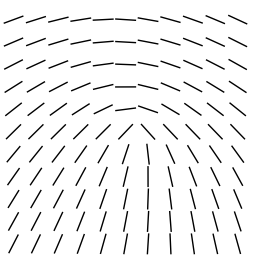

(a) Loop I

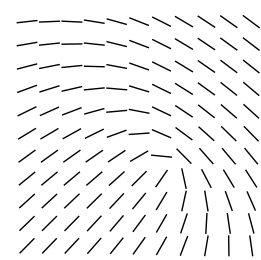

(b) Loop II

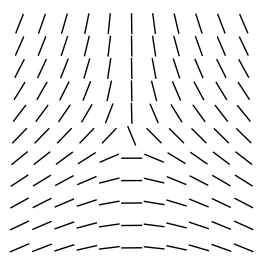

(c) Delta
Fig. 3. Half-phase portrait of (a) center (concentric circles); (b) spiral; (c) saddle.

Firstly, the orientation angle is defined within $[0, \pi)$ which has an intrinsic discontinuity from $\pi$ to 0 while the differential equations assume continuity. Secondly, the linear model cannot give direct description for loop-type and delta-type SPs. Finally, the linear model cannot represent multiple SPs in a local region.

The first two problems can be resolved by modeling the squared OF. The squared OF is associated with doubled orientation angles [10]. The phase change thus becomes continuous. To address the last problem, we propose an elliptic curve model as follows.

As shown in Figure 2, a whorl or twin-loop SP can be directly modeled by the linear system (1). From the trigonometric double-angle formula, the associated squared OF can be modeled by

$$
\begin{aligned}
\cos 2 \theta(x, y) & =2 \cos ^{2} \theta(x, y)-1 \\
& =2 F(x, y)^{2}-1 \\
\sin 2 \theta(x, y) & =2 \cos \theta(x, y) \sin \theta(x, y) \\
& =2 F(x, y) G(x, y) .
\end{aligned}
$$

Now the linear system is squared to two elliptic functions as shown in (2). With matrix notation, the estimated phases can also be written as

$$
\begin{aligned}
& \cos 2 \hat{\theta}(x, y)=2 \mathbf{p}^{T} \alpha \alpha^{T} \mathbf{p}-1 \\
& \sin 2 \hat{\theta}(x, y)=2 \mathbf{p}^{T} \alpha \beta^{T} \mathbf{p},
\end{aligned}
$$

where $\mathbf{p}=[x, y, 1]^{T}$ is the variable vector and $\alpha=[a, b, e]^{T}$, $\beta=[c, d, f]^{T}$ are parameters for $F$ and $G$ respectively.

The elliptic model can be optimized by minimizing squared errors between the estimation and the observation over the local singular region $D$. Let

$$
\begin{aligned}
& t_{c}(x, y)=\frac{1}{2} \cos 2 \theta(x, y)+\frac{1}{2}, \\
& t_{s}(x, y)=\frac{1}{2} \sin 2 \theta(x, y) .
\end{aligned}
$$

be the training data on the observation of $\theta$ at each locus $(x, y)$. Then the optimization is obtained by

$$
\begin{aligned}
& \min _{\alpha} \sum_{D}\left\|\mathbf{p}^{T} \alpha \alpha^{T} \mathbf{p}-t_{c}(x, y)\right\|^{2}, \\
& \min _{\alpha, \beta} \sum_{D}\left\|\mathbf{p}^{T} \alpha \beta^{T} \mathbf{p}-t_{s}(x, y)\right\|^{2}
\end{aligned}
$$

with respect to the parameters $\alpha$ and $\beta$.

Standard algorithms, such as the Levenberg-Marquardt method, for nonlinear least square optimizations can be used to solve (5). The whorl and twin-loop can be reconstructed directly from the linear system (1) defined by the parameters $\alpha$ and $\beta$. For loop and delta, the OF is represented by the half phase portrait of (3).

Further consider the proposed elliptic model in (3). If we let $\mathbf{B}_{c}=\alpha \alpha^{T}, \mathbf{B}_{s}=\alpha \beta^{T}$ and omit the constant and scales, then (3) is reduced to

$$
\begin{aligned}
\cos 2 \hat{\theta}(x, y) & =\mathbf{p}^{T} \mathbf{B}_{c} \mathbf{p} \\
\sin 2 \hat{\theta}(x, y) & =\mathbf{p}^{T} \mathbf{B}_{s} \mathbf{p}
\end{aligned}
$$

which becomes a pair of second-order bivariate polynomial functions. Thus it can be considered as an approximation to the proposed elliptic model.

The main benefit of using the polynomial approximation is in that the tedious nonlinear least square optimization can be replaced by a fast and more reliable linear least square method. We report our results of preliminary experiments in the next section.

\section{PERFormance Evaluation}

The above elliptic curve model is proposed for estimating local orientations near singular points. For estimation of the overall orientation filed, it is combined with a fourthorder polynomial that provides a description for the global smoothness. The combination process is similar as in [7]. That is, a weighing function is introduced to insure that a close orientation estimate follows the elliptic model if it is close to a singular point or follows the global polynomial model if it is otherwise. The boundary of the separation depends on experimental experience.

Figure 4 gives a concrete example to show the estimation effects of different approaches used for orientation estimation. The estimated results are superimposed on the same fingerprint image. For more objective evaluation of high curvature estimation in the SP regions, the core of whorl and delta are manually detected for the fingerprint and marked by a dot and a triangle respectively in the image.

Figure 4(a) is from an enhanced gradient-based method [2] that estimates orientations from gray intensity changes of the fingerprint. Since it is directly from the image, the coarse OF is used as training input to the OF models used in subsequent figures. One can see that Figure 4(a) is coarse due to the noise-sensitive nature of gradient-based methods [1]. Although the averaging process can help to reduce noise effects, it also tends to level out the important high curvature information. In our experiments, we are keen on preserving high curvature information thus tradeoff is given by sacrificing noise resilience in coarse estimates.

The OF displayed in Figure 4(b) is reconstructed from a fourth-order bivariate polynomial model. One can see that the resulting OF estimates are fairly smooth in a global sense but the topology pattern was distorted at the whorl center. 


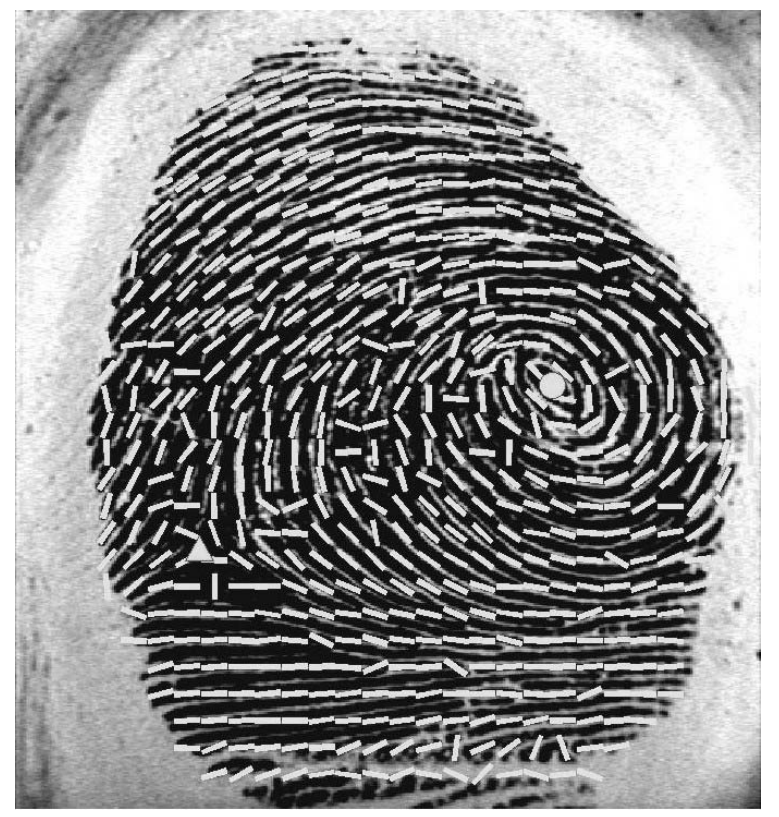

(a) Gradient-based

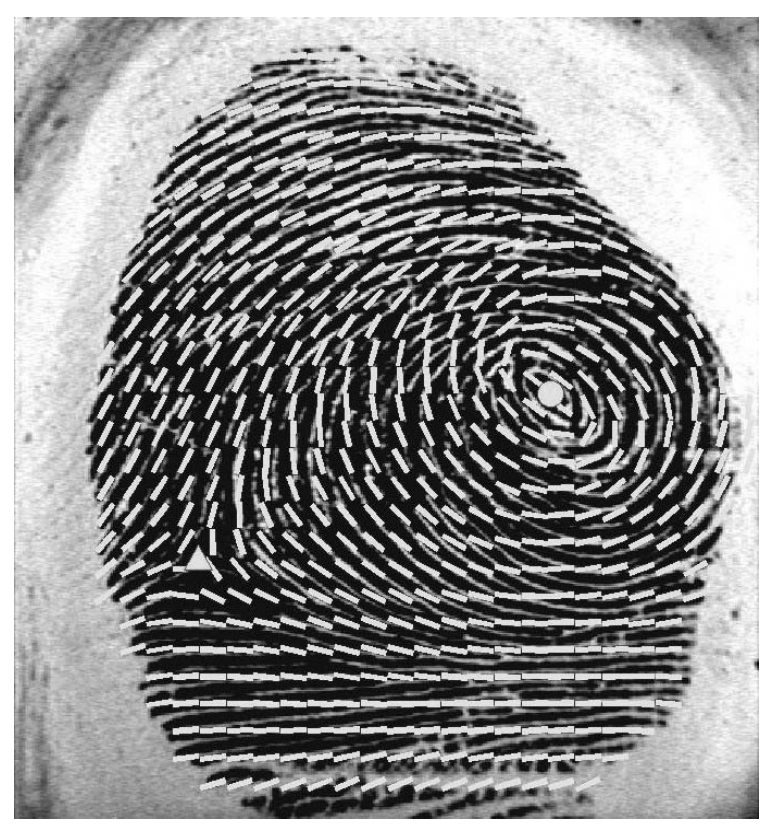

(c) Global polynomial + Point-charge

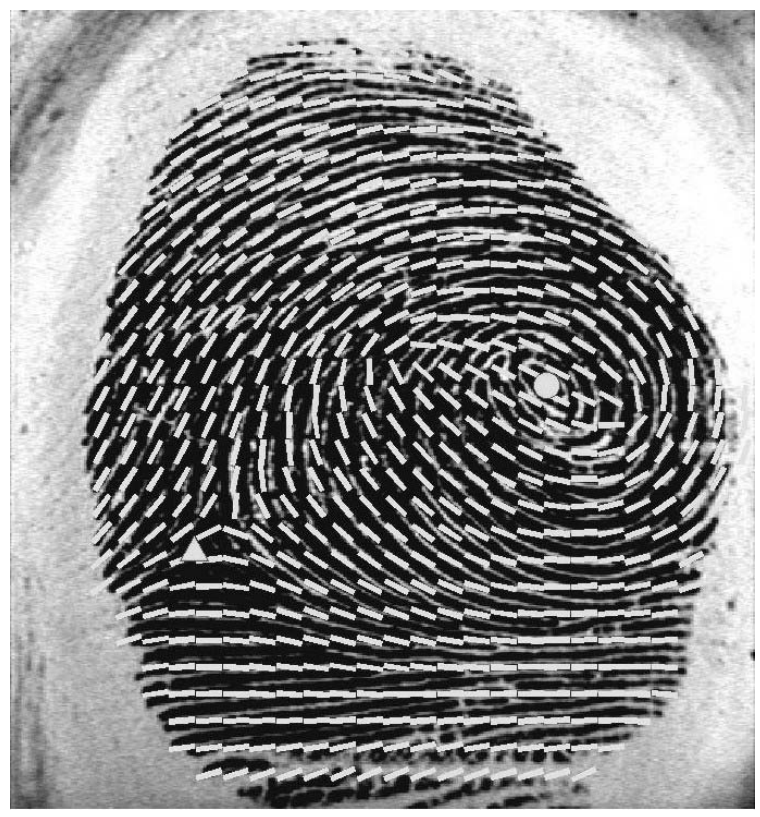

(b) Global polynomial

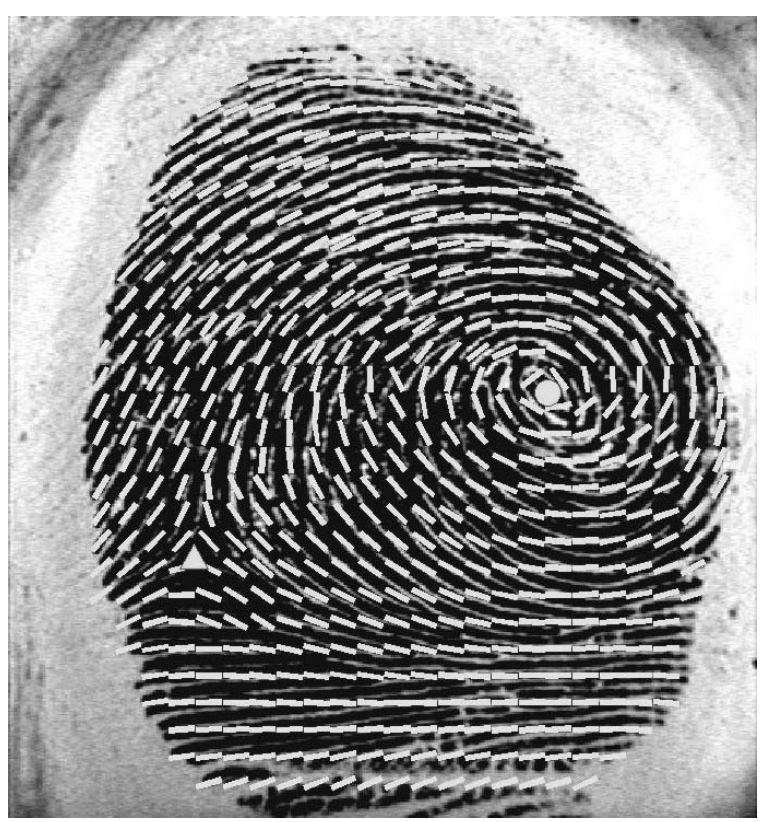

(d) Global polynomial + Proposed elliptic curve

Fig. 4. OF estimates from different approaches on the same fingerprint. The whorl and delta are marked with $\bigcirc$ and $\triangle$ respectively.

Figure 4(c) replaced the distorted estimates near the SP regions at the whorl and delta with those from the point-charge model [7]. One can see that the estimation for high curvature is improved, but the interpretation around the core, especially to the left, does not coincide with the original trends of ridge flows as shown in the background.

Figure 4(d) demonstrates orientation estimates from the proposed elliptic model in combination with the global fourthorder polynomial model. Comparing with results shown in the other figures, the proposed model gives better description in the singular regions where ridges are bent with high curvature.

Figure 5 displays another set of comparison for the local orientation estimation on singular patterns. The estimated results based on the four comparing methods are again superimposed 


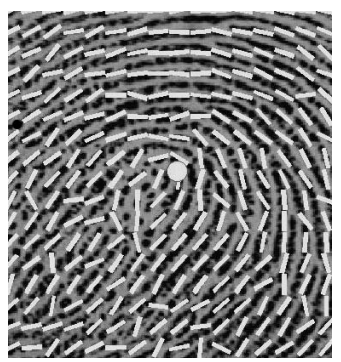

(a) Gradient-based

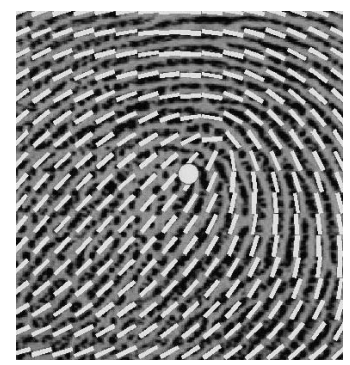

(b) Global polynomial

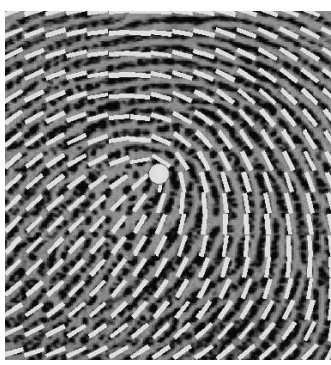

(c) Point-charge

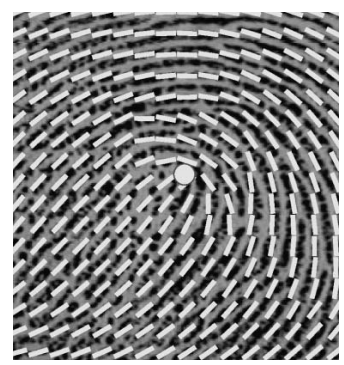

(d) Elliptic curve

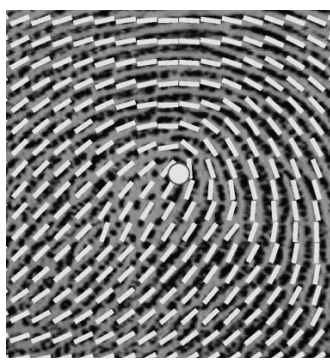

(e) Local polynomial

Fig. 5. OF estimates from different approaches on a fingerprint segment with high curvature. The embedded loop-like SP is marked with $\bigcirc$.

on the tested fingerprint segments. The loop-type SP is also manually identified and marked.

Figure 5(b) is the corresponding section in the overall OF constructed from the global four-order polynomial model. On the other hand, figure 5(c)-5(e) are estimation results constructed from different local orientation models around the loop of SP. That is, the estimation is only based on training data in the local region. We see that the results from those local orientation models have gained improvements against their coarse orientation input as shown in figure 5(a), and that from the global polynomial model. In particular, the local second order polynomial approximation, although slightly less accurate than the elliptic curve model at the SP position, coincides with most of the highly curved ridge trends.

From our experiments, it seems that the second-order polynomial function is a good approximation to the elliptic curve model, which gives satisfactory performance for the orientation estimation of singular pattern with high curvature. Table I lists the computational time required by solving the compared local orientation models for the above fingerprint segment.

\section{TABLE I}

COMPUTATIONAL TIME REQUIRED BY SOLVING THE LOCAL ORIENTATION MODELS FOR A FINGERPRINT SEGMENT.

\begin{tabular}{c|c|c|} 
point-charge & Elliptic-curve & 2nd-order polynomial \\
\hline 0.078 seconds & 0.152 seconds & 0.032 seconds \\
\hline
\end{tabular}

The computational time listed above is obtain from the same computing environment on a Pentium $43.0 \mathrm{GHz}$ machine with a Matlab implementation.

From Table I, we see that the second-order polynomial approximation to the elliptic curve model possesses obvious computational advantage over the other two local orientation models. This is because it only requires a fast linear least square for the optimization process. Therefore, we adopt it in placement of the proposed combination model for evaluating the statistical performance.

As we have introduced in Section I, the estimation of orientation fields (OFs) is a fundamental process in fingerprint authentication systems (FAS). An accurate OF extraction has a great impact on the final fingerprint matching performance. Therefore, the comparing OF extraction algorithms will be tested on the same FAS platform by replacing them with the OF estimation module in the system respectively.

The FAS uses minutiae matching to verify input fingerprint queries. The system performance is assessed by two matching indices: false acceptance rate (FAR) and false rejection rate (FRR). The FAR index is defined as the general percentage of an imposter being falsely accepted by the system, while the FRR index is the probability of a genuine user being falsely rejected.

We run our experiments on a public database used in the FVC2002 contest [1], which contains 800 live-scanned fingerprints from 100 fingers each gives 8 different impressions. All the images are captured by an optical sensor with a resolution of 500dpi.

In our experiments, each fingerprint is mesh matched with one another in the database, which results in a total $\frac{1}{2} \times(799 \times$ $800)=319,600$ pairs of comparison. Among them, $\frac{1}{2} \times(7 \times$ $800)=2800$ are genuine pairs (i.e. the two in a pair are from the same fingers $)$ and $\frac{1}{2} \times(8 \times 99 \times 800)=316,800$ are imposter pairs (i.e. from different fingers).

The system performance is usually reported by a Receiver Operating Characteristics (ROC) curve that plots the two indices FAR versus FRR at various matching thresholds. Figure 6 depicts the ROC curves for the authentication system based on the comparing OF estimation methods.

As shown in Figure 6, the accuracy of an OF estimation can largely affect the system's final matching performance. All the model based methods have improved from the coarse OF directly computed from the gray scale image. Among the three model-based approaches, the proposed model achieves the best gain.

\section{CONCLUSIONS}

Orientation estimation is important in fingerprint authentication systems. It directly affects the end performance of system false acceptance rate and false rejection rate. An accurate orientation model helps in improving orientation estimation as well as other important fingerprint applications such as indexing. 


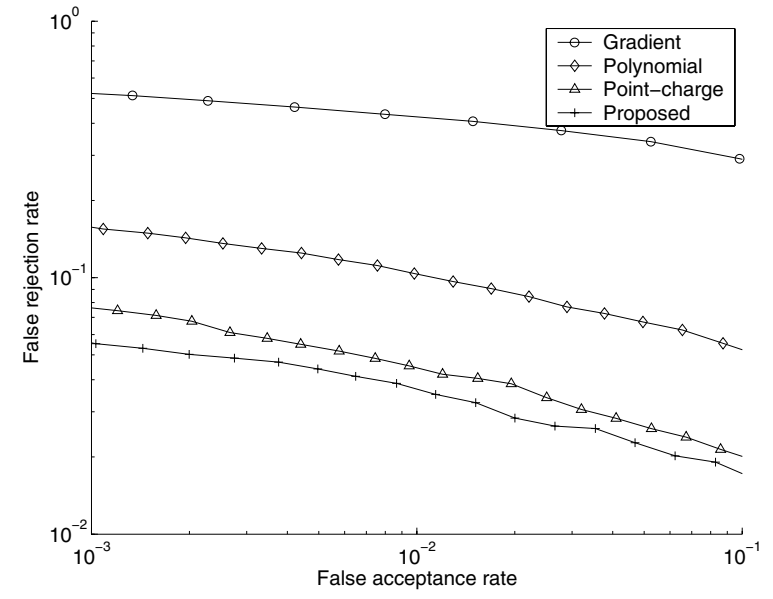

Fig. 6. ROC with different OF extraction algorithms.

Fingerprints often possess both smooth ridge flows and singular regions with high curvature. It is difficult to accommodate these different patterns in a single mathematical description. A combination approach can be considered to address the problem. In this paper, we investigated different local orientation models in order to improve the local orientation estimates near the singular points. We first proposed an elliptic curve model derived from a linear system of differential equations. A more general second-order polynomial model is then considered for the approximation of the elliptic curve model in order to reduce the computational complexity.

We show that the second-order polynomial approximation, although slightly less accurate in representing the singular point locations, possesses an obvious computation advantage in modeling the local singular region. Therefore, we adopt it in placement of the local orientation model in the combination approach.

The combination of different polynomial models, globally with a higher order and locally with second order, enables a global topology description for both smooth patterns and the critical high curvature patterns. The proposed combination approach has been evaluated on real fingerprint images in comparison with other existing methods. Our preliminary results show that the proposed approach can improve the false acceptance rate and false rejection rate of fingerprint authentication systems.

However, in general, the combination approach has an inherent problem of uncertainty as it relies heavily on the accurate detection of singular points which is by itself not a trivial task. The performance of such a method may degrade largely if the detected singular points are faulty. Moreover, there seems to have no clear rules on the partition of patching areas for the combinational modeling. The actual practice usually depends on experimental experience. Hence, there is a need to explore new framework that can systematically construct a consistent description of the entire orientation field. We have make some progress along this direction [13].

\section{ACKNOWLEDGEMENT}

This work is financially supported by the ARC Linkage Project LP0455324.

The authors would also like to thank Professor Zhou and his assistants of the Tsing Hua University for their kind supports on our implementation of their combination model [7].

\section{REFERENCES}

[1] D. Maltoni, D. Maio, A. K. Jain, and S. Prabhakar, Handbook of fingerprint recognition. New York: Springer-Verlag, 2003.

[2] Y. Wang, J. Hu, and F. Han, "Enhanced gradient based algorithm for the estimation of fingerprint orientation fields," Applied Mathematics And Computation, online Aug 2006.

[3] A. Jain, L. Hong, and R. Bolle, "On-line fingerprint verification," IEEE Trans. Pattern Anal. Machine Intell., vol. 19, no. 4, pp. 302-314, Apr 1997.

[4] Y. Wang, J. Hu, and H. Schroder, "A gradient based weighted averaging method for estimation of fingerprint orientation fields," in Digital Image Computing: Techniques and Applications, Cairns, Australia, Dec 2005.

[5] B. Sherlock, D. Monro, and K. Millard, "Fingerprint enhancement by directional fourier filtering," in IEE Proc. Vision, Image and Signal Processing, vol. 141, no. 2, Apr 1994, pp. 87-94.

[6] S. Dass, "Markov random field models for directional field and singularity extraction in fingerprint images," IEEE Trans. Image Processing, vol. 13, no. 10, pp. 1358-1367, 2004.

[7] J. Zhou and J. Gu, "A model-based method for the computation of fingerprints orientation field," IEEE Trans. Image Processing, vol. 13, no. 6, pp. 821-835, 2004.

[8] W. Yau, J. Li, and H. Wang, "Nonlinear phase portrait modeling of fingerprint orientation," in IEE Proc. Control, Automation, Robotics and Vision Conference, vol. 2, Dec 2004, pp. 1262-1267.

[9] R. Ford and R. Strickland, "Nonlinear phase portrait models for oriented textures," in Proceedings of Computer Vision and Pattern Recognition. IEEE computer society, June 1993, pp. $644-645$.

[10] M. Kass and A. Witkin, "Analyzing oriented patterns," Computer Vision, Graph and Image Processing, vol. 37, no. 3, pp. 362-385, Mar 1987.

[11] A. Rao and R. Jain, "Computerized flow field analysis: oriented texture fields," IEEE Trans. Pattern Anal. Machine Intell., vol. 14, no. 7, pp. 693-709, Jul 1992.

[12] L. Perko, Differential equations and dynamical systems. New York: Springer-Verlag, 1991.

[13] Y. Wang, J. Hu, and D. Phillips, "A fingerprint orientation model based on $2 \mathrm{~d}$ fourier expansion (fomfe) and its application to singular-point detection and fingerprint indexing," IEEE Trans. Pattern Anal. Machine Intell., to appear in May 2007. 